\title{
SUBALGEBRAS OF DOUGLAS ALGEBRAS
}

\author{
KEVIN CLANCEY AND WAYNE CUTRER
}

\begin{abstract}
A closed subalgebra $\mathscr{A}$ of $L^{\infty}$ is called a Douglas algebra in case $\mathscr{A}$ is an algebra generated by $H^{\infty}$ and a set of inverses of inner functions. It is shown that if the Douglas algebra $\mathscr{A}$ contains properly $H^{\infty}+C$, then there is another Douglas algebra $\mathscr{A}^{\prime}$ such that $H^{\infty}+C \varsubsetneqq \mathscr{A}^{\prime} \varsubsetneqq \mathscr{A}$. Some results on subalgebras are also given for algebras generated by $H^{\infty}$ and a function of the form $f \bar{B}$, where $f$ is in $H^{\infty}$ and $B$ is an infinite Blaschke product.
\end{abstract}

Let $H^{\infty}$ be the algebra of bounded functions analytic in the unit disk $D$. The notation $L^{\infty}$ will denote the algebra of bounded Lebesgue measurable functions on the unit circle. The space of continuous functions on the unit circle will be denoted by $C$. This note is concerned with closed subalgebras $\mathscr{A}$ of $L^{\infty}$ that contain $H^{\infty}$. The maximal ideal space of the algebra $\mathscr{A}$ will be denoted by $M_{\mathscr{A}}$.

A closed subalgebra $\mathscr{A}$ of $L^{\infty}$ is called a Douglas algebra in case $\mathscr{A}$ is the algebra $H^{\infty}[\Sigma]$ generated by $H^{\infty}$ and a set $\Sigma$ of inverses of inner functions. In the special case where $\Sigma$ contains the inverse of a single inner function $\varphi$ the notation $H^{\infty}[\bar{\varphi}]$ is used for the Douglas algebra $H^{\infty}[\Sigma]$.

Douglas [2] has conjectured that every closed algebra $\mathscr{A}$ satisfying $H^{\infty} \subset \mathscr{A} \subset L^{\infty}$ is a Douglas algebra. A recent discussion of this problem is contained in Sarason [5].

Douglas has also asked whether every subalgebra $\mathscr{A}$ of $L^{\infty}$ satisfying $H^{\infty}+C \varsubsetneqq \mathscr{A}$ contains properly a subalgebra $\mathscr{A}^{\prime}$ satisfying $H^{\infty}+C \varsubsetneqq \mathscr{A}^{\prime}$. We give an affirmative answer to this question when $\mathscr{A}$ is a Douglas algebra.

Our main result is:

TheOREM 1. Let $\mathscr{A}=H^{\infty}[\Sigma]$ be a Douglas algebra which properly contains $H^{\infty}+C$. There exists another Douglas algebra $\mathscr{A}^{\prime}$ such that $H^{\infty}+C \subsetneq \mathscr{A}^{\prime} \subsetneq \mathscr{A}$.

The above theorem makes it obvious that Douglas's second question is weaker than the first.

Received by the editors September 8, 1972 and, in revised form, November 17, 1972. AMS (MOS) subject classifications (1970). Primary 46J10.

(c) American Mathematical Society 1973 
1. Proof of Theorem 1. Fortunately, if a subalgebra of $L^{\infty}$ properly contains $H^{\infty}$, then it contains $H^{\infty}+C$ (see, Hoffman [4, p. 193]). Therefore, if $\varphi$ is a nonconstant inner function, then $H^{\infty}[\bar{\varphi}]$ contains $H^{\infty}+C$. Clearly, $H^{\infty}[\bar{\varphi}]=H^{\infty}+C$ whenever $\varphi$ is a nonconstant finite Blaschke product. The converse of this last statement is true; that is, if $\varphi$ is inner and $H^{\infty}[\bar{\varphi}]=H^{\infty}+C$, then $\varphi$ must be a nonconstant finite Blaschke product. To see this, observe that $M_{H^{\infty}+C}=M_{H^{\infty}} \sim D$ (Hoffman [4, p. 207, Exercise 1]), and when $H^{\infty}[\bar{\varphi}]=H^{\infty}+C$, then $|\hat{\varphi}(\gamma)|=1$ for every $\gamma$ in $M_{H^{\infty}+C}$ (here, $\hat{\varphi}$ denotes the Gelfand transform of $\varphi$ ). This is impossible since if $\varphi$ is not a finite Blaschke product, then there are always points $\gamma$ in $M_{H^{\infty}+C}$ where $\hat{\varphi}(\gamma)=0$.

A theorem of Frostman (see, e.g. Hoffman [4, p. 175]) permits one to conclude that for some small $\lambda$ in $D$ the function $S_{\lambda}=(\lambda-\varphi)(1-\bar{\lambda} \varphi)^{-1}$ is a Blaschke product. This result and the remarks of the preceding paragraph show that it suffices to prove Theorem 1 in the case where the algebra $\mathscr{A}$ is $H^{\infty}[\bar{B}]$; here, $B$ is an infinite Blaschke product.

Douglas and Rudin [3] prove that, for $\varphi$ inner, $M_{H^{\infty}[\bar{\varphi}]}$ is the set $K_{\varphi}=\left\{\gamma:|\hat{\varphi}(\gamma)|=1, \gamma \in M_{H^{\infty}}\right\}$. Since the homomorphisms of $H^{\infty}$ have unique Hahn-Banach (norm-preserving) extensions to linear functionals on $L^{\infty}$, it is easily seen that whenever the algebras $\mathscr{A}$ and $\mathscr{A}^{\prime}$ satisfy $H^{\infty} \subset \mathscr{A}^{\prime} \subset \mathscr{A} \subset L^{\infty}$, then $M_{\mathscr{A}} \subset M_{\mathscr{A}^{\prime}}$ (see, Hoffman [4, p. 182]).

If the Blaschke product $B_{0}$ divides $B$, then $H^{\infty}\left[\bar{B}_{0}\right] \subset H^{\infty}[\bar{B}]$. In order to complete the proof of Theorem 1 it suffices to show that for every infinite Blaschke product $B$ there is an infinite Blaschke product $B_{0}$ dividing $B$ such that $K_{B} \subsetneq K_{B_{0}}$. The key to showing this is the following lemma concerning "strong" exponential sequences.

Lemma 1. Let $\left\{z_{k}\right\}_{k=1}^{\infty} \subset D$ and assume $\lim \sup _{k \rightarrow \infty}\left|z_{k}\right|=1$. Then there exists a subsequence $\left\{z_{n_{j}}\right\}_{j=1}^{\infty}$ such that

$$
\inf _{k}\left[\prod_{j=1 ; j \neq k}^{\infty}\left|\frac{z_{n_{k}}-z_{n_{j}}}{1-\bar{z}_{n_{j}} z_{n_{k}}}\right|^{j}\right]>0 .
$$

Proof. Let $0<c<1$ and let $\left\{p_{k}\right\}_{k=1}^{\infty}$ be any infinite strictly increasing sequence of positive integers. Set $z_{n_{1}}=z_{1}$ and inductively choose $\left\{z_{n_{k}}\right\}_{k=1}^{\infty}$ such that

$$
1-\left|z_{n_{k+1}}\right|<c^{p_{k+1}}\left[1-\left|z_{n_{k}}\right|\right] .
$$

For $j>k, 1-\left|z_{n_{j}}\right|<c^{p_{j}+\cdots+p_{k+1}}\left[1-\left|z_{n_{k}}\right|\right]$, which implies

Further,

$$
\left|z_{n_{j}}\right|-\left|z_{n_{k}}\right| \geqq\left[1-c^{p_{j}+\cdots+p_{k+1}}\right]\left[1-\left|z_{n k}\right|\right] \text {. }
$$

$$
1-\left|z_{n_{j}}\right|\left|z_{n_{k}}\right| \leqq 1-\left|z_{n_{j}}\right|+1-\left|z_{n_{k}}\right| \leqq\left[1+c^{p_{j}+\cdots+p_{k}}\right]\left[1-\left|z_{n_{k}}\right|\right] .
$$


Using the estimate $|\alpha-\beta| /|1-\bar{\alpha} \beta| \geqq(|\alpha|-|\beta|) /(1-|\alpha||\beta|)$, for $\alpha, \beta \in D$, it follows that

$$
\begin{aligned}
\prod_{j>k}\left\{\frac{\left|z_{n_{k}}-z_{n}\right|}{\left|1-\bar{z}_{n_{j}} z_{n_{k}}\right|}\right\}^{j} & \geqq \prod_{j>k}\left\{\frac{\left|z_{n_{j}}\right|-\left|z_{n_{k}}\right|}{1-\left|z_{n_{j}}\right|\left|z_{n_{k}}\right|}\right\}^{j} \\
& \geqq \prod_{j>k}\left\{\frac{1-c^{p_{j}+\cdots+p_{k}}}{1+c^{p_{j}+\cdots+p_{k}}}\right\}^{j} \geqq \prod_{j>k}\left\{\frac{1-c^{p_{j}}}{1+c^{p_{j}}}\right\}^{j} .
\end{aligned}
$$

For $j<k$, one can obtain similarly

$$
\prod_{j<k}\left\{\frac{\left|z_{n_{k}}-z_{n_{j}}\right|}{\mid 1-\bar{z}_{n_{j}} z_{n_{k}}}\right\}^{j} \geqq \prod_{j<k}\left\{\frac{1-c^{p_{j}}}{1+c^{p_{j}}}\right\}^{j} .
$$

Next select an increasing sequence $\left\{p_{j}\right\}_{j=1}^{\infty}$ of nonnegative integers such that $\left(1-c^{p_{j}}\right) /\left(1+c^{p_{j}}\right) \geqq\left\{\left(1-c^{j}\right) /\left(1+c^{j}\right)\right\}^{1 / j}$. If the $z_{n_{j}}$ are chosen in accordance with (2) using this sequence $\left\{p_{j}\right\}$, then

$$
\inf _{k} \prod_{j \neq k}\left\{\frac{\left|z_{n_{k}}-z_{n_{j}}\right|}{\left|1-\bar{z}_{n_{j}} z_{n_{k}}\right|}\right\}^{j} \geqq \prod_{j=1}^{\infty} \frac{1-c^{j}}{1+c^{j}}>0 .
$$

This completes the proof.

The proof of Lemma 1 is fashioned after Newman's proof that an exponential sequence is an interpolating sequence (Hoffman $[4$, p. 203]).

Now we complete the proof of Theorem 1 .

Select a subsequence $\left\{z_{n_{j}}\right\}_{j=1}^{\infty}$ of the zeros of $B$ satisfying (1) and $\sum_{k=1}^{\infty} k\left[1-\left|z_{n_{k}}\right|\right]<\infty$. For $k=1,2, \cdots$, let

$$
Q_{k}(z)=\frac{z_{n k}}{\left|z_{n_{k}}\right|} \frac{z-z_{n k}}{1-\bar{z}_{n k} z}
$$

and set $B^{\prime}=\prod_{k=1}^{\infty} Q_{k}$. Factor $B^{\prime}=B_{e} B_{0}$, where $B_{e}=\prod_{p=1}^{\infty} Q_{2 p}$ and $B_{0}=$ $\prod_{p=1}^{\infty} Q_{2 p-1}$. Finally form $\widetilde{B}_{0}=\prod_{p=1}^{\infty}\left[Q_{2 p-1}\right]^{2 p-1}$. Let $\gamma$ be a point in $M_{H^{\infty}}^{\infty} \sim D$ and in the closure of $\left\{z_{n_{2 p}}\right\}_{p=1}^{\infty}$. Since (1) holds, then $\left|\tilde{B}_{0}^{\hat{0}}(\gamma)\right|>0$ and, clearly, $\left|\hat{B}_{0}(\gamma)\right| \geqq\left|\widetilde{B}_{0}^{\wedge}(\gamma)\right|$; moreover, $\hat{B}^{\prime}(\gamma)=0$.

Fix $q>1$ and define

$$
C_{q}=\prod_{j=q+1}^{\infty}\left(Q_{2 j-1}\right)^{2(j-q)} \text { and } P_{q}=\prod_{j=1}^{q}\left(Q_{2 j-1}\right)^{2(q-j)} .
$$

From the identity $B_{0}^{2 q-1} C_{q}=\widetilde{B}_{0} P_{q}$, it follows that

$$
\hat{B}_{0}^{2 q-1}(\gamma) \hat{C}_{q}(\gamma)=\widetilde{B}_{0}^{\wedge}(\gamma) \hat{P}_{q}(\gamma) .
$$

Then $\left|\hat{B}_{0}(\gamma)\right|^{2 q-1} \geqq\left|\widetilde{B}_{0}^{\hat{0}}(\gamma)\right|>0$ and, therefore, $\left|\hat{B}_{0}(\gamma)\right| \geqq 1$. It follows that $M_{H^{\infty}[\bar{B}]} \subsetneq M_{H^{\infty}\left[\bar{B}_{0}\right]}$ and, therefore, $H^{\infty}+C \subsetneq H^{\infty}\left[\bar{B}_{0}\right] \subsetneq H^{\infty}[\bar{B}]$. This ends the proof of Theorem 1 . 
2. In this section we will briefly discuss algebras generated by $H^{\infty}$ and a function $f \bar{B}$ where $f$ is in $H^{\infty}$ and $B$ is an infinite Blaschke product. A major obstacle to studying these algebras is a lack of working knowledge of whether the function $f \bar{B}$ belongs to $H^{\infty}+C$ (Sarason in [6, Theorem 2] has given necessary and sufficient conditions for $f \bar{B}$ to be in $H^{\infty}+C$ in terms of the compression of the operator of multiplication by $f$ onto the star invariant $\left.H^{2} \ominus B H^{2}\right)$. We will consider a fairly obvious case.

Let $\left\{a_{k}\right\}_{k=1}^{\infty}$ be the zeros of $B$ and assume lim sup $\left|f\left(a_{k}\right)\right| \neq 0$. The function $f \bar{B}$ is not in $H^{\infty}+C$. To see this, observe that if $\left|f\left(a_{k_{n}}\right)\right| \geqq \delta>0$ on the infinite subsequence $\left\{a_{k_{n}}\right\}_{n=1}^{\infty}$ of the zeros of $B$, then for every $\gamma$ in the closure of $\left\{a_{k_{n}}\right\}_{n=1}^{\infty}$ in $M_{H^{\infty}}$ we have $|\hat{f}(\gamma)| \geqq \delta>0$. If $f \bar{B}$ is in $H^{\infty}+C$, then $f \bar{B}=g+p$ where $g$ is in $H^{\infty}$ and $p$ is in $C$. Since $|B|=1$, then $f=B g+B p$. It follows that $\hat{f}$ must vanish on $M_{H^{\infty}}+C$ where $\hat{B}$ does, contradicting the fact that $|\hat{f}(\gamma)| \geqq \delta$ for some $\gamma$ where $\hat{B}$ vanishes.

We can prove the following analogue of Theorem 1 for the algebras $H^{\infty}[f \bar{B}]$.

Proposition 1. Let $B$ be an infinite Blaschke product with zero sequence $\left\{a_{k}\right\}_{k=1}^{\infty}$. Let $f$ be in $H^{\infty}$ and assume $\lim \sup \left|f\left(a_{k}\right)\right| \neq 0$, then there is a subalgebra of $H^{\infty}[f \bar{B}]$ of the form $H^{\infty}\left[f \bar{B}_{0}\right]$, where $B_{0}$ is a Blaschke product dividing $B$, satisfying $H^{\infty}+C \subsetneq H^{\infty}\left[f \bar{B}_{0}\right] \subsetneq H^{\infty}[f \bar{B}]$.

Proof. Clearly if $B^{\prime}$ is a Blaschke product dividing $B$, then $H^{\infty}\left[f \bar{B}^{\prime}\right] \subset$ $H^{\infty}[f \bar{B}]$ and we can, therefore, assume that $\lim \inf \left|f\left(a_{k}\right)\right| \neq 0$. It is possible (by the techniques in $\S 1$ ) to factor $B=B_{0} B_{e}$ such that $B_{0}$ and $B_{e}$ are infinite Blaschke products and such that $\lim _{k}\left|B_{0}\left(c_{k}\right)\right|=1$, where $\left\{c_{k}\right\}_{k=1}^{\infty}$ is an infinite subsequence of the zeros of $B_{e}$.

The following inclusions exist:

$$
\begin{aligned}
H^{\infty}\left[\bar{B}_{0}\right] & \subsetneq H^{\infty}[\bar{B}] \\
\cup & \cup \\
H^{\infty}+C \varsubsetneqq H^{\infty}\left[f \bar{B}_{0}\right] & \subset H^{\infty}[f \bar{B}] .
\end{aligned}
$$

Let $\gamma$ be a point in $M_{H^{\infty}+C}$ and in the closure of $\left\{c_{k}\right\}_{k=1}^{\infty}$. If $\gamma$ is in $M_{H^{\infty}[f B]}$, then $(f \bar{B})^{\wedge}(\gamma) \hat{B}(\gamma)=(f \bar{B} B)^{\wedge}(\gamma)=\hat{f}(\gamma)$ and since $\hat{B}(\gamma)=0$ and $\hat{f}(\gamma) \neq 0$ a contradiction results. It follows that $\gamma$ is not in $M_{H^{\infty}[f B]}$.

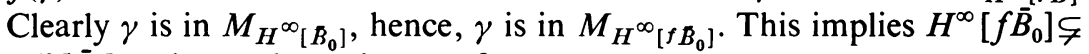
$H^{\infty}\left[\bar{B}_{0}\right]$ and completes the proof.

REMARKS. If the zero set $\left\{a_{k}\right\}_{k=1}^{\infty}$ of $B$ is an interpolating sequence, then it is possible to combine the results of Clark [7, Theorem 2.1] and Sarason [6, Theorem 2] to obtain that $f \bar{B}$ is in $H^{\infty}+C$ if and only if $\lim f\left(a_{k}\right)=0$. Moreover, in this case the zero set of $\hat{B}$ is the closure of 
$\left\{a_{k}\right\}_{k=1}^{\infty}$ in $M_{H^{\infty}}($ Hoffman $[4$, p. 206]). It follows that if $\lim \inf \left|f\left(a_{k}\right)\right| \neq 0$,

then $H^{\infty}[f \bar{B}]=H^{\infty}[\bar{B}]$. To see this let $\gamma$ be in the zero set of $\hat{B}$. If $\gamma$ is multiplicative on $H^{\infty}[f \bar{B}]$, then $(f \bar{B})^{\wedge} \hat{B}(\gamma)=\hat{f}(\gamma)$ and since $\hat{f}(\gamma) \neq 0$ and $\hat{B}(\gamma)=0$ a contradiction results. Therefore, $B$ does not vanish on $M_{H^{\infty}[f \bar{B}]}$ and it follows that $\bar{B}$ is in $H^{\infty}[f \bar{B}]$.

A similar argument can be used to show that if $f \bar{B}$ is not in $H^{\infty}+C$ and the zeros of $B$ form an interpolating sequence, then $H^{\infty}[f \bar{B}]$ contains a Douglas algebra different from $H^{\infty}+C$. The authors have been unable to decide, in this case, whether $H^{\infty}[f \bar{B}]$ is a Douglas algebra.

ADDED IN PROOF. A recent result of Sarason can be used to show that any algebra properly containing $H^{\infty}+C$ contains an algebra generated by $H^{\infty}$ and the inverse of an infinite Blaschke product. This result, along with the results in this paper, implies an affirmative answer to Douglas' second question.

\section{REFERENCES}

1. R. G. Douglas, Banach algebra techniques in the theory of Toeplitz operators (preprint).

2. - On the spectrum of Toeplitz and Weiner-Hopf operators, Abstract Spaces and Approximation, Birkhaüser, Basel, 1969, pp. 53-66. MR 41 \#4274.

3. R. G. Douglas, and W. Rudin, Approximation by inner functions, Pacific J. Math. 31 (1969), 313-320. MR 40 \#7814.

4. K. Hoffman, Banach spaces of analytic functions, Prentice-Hall, Englewood Cliffs, N.J., 1962, MR 24 \#A2844.

5. D. Sarason, Algebras of functions on the unit circle (preprint).

6. - , Generalized interpolation in $H^{\infty}$, Trans. Amer. Math. Soc. 127 (1967), 179-203. MR 34 \#8193.

7. D. N. Clark, On interpolating sequences and the theory of Hankel and Toeplitz matrices, J. Functional Analysis 5 (1970), 247-258. MR 40 \#7836.

Department of Mathematics, University of Georgia, Athens, Georgia 30602 